\title{
Association between lipoprotein(a) concentration and the risk of stroke in the Chinese Han population: a retrospective case-control study
}

\author{
Hanhui Fu ${ }^{1}$, Dingding Zhang ${ }^{2}$, Rui Zhu ${ }^{3}$, Liying Cui ${ }^{1}$, Ling Qiu ${ }^{4}$, Songbai Lin ${ }^{5}$, Bin Peng ${ }^{1}$ \\ ${ }^{1}$ Department of Neurology, Peking Union Medical College Hospital, Chinese Academy of Medical Sciences, Beijing 100730, China; ${ }^{2}$ Central \\ Research Laboratory, Peking Union Medical College Hospital, Chinese Academy of Medical Sciences and Peking Union Medical College, Beijing \\ 100730, China; ${ }^{3}$ Department of Medical Record, ${ }^{4}$ Department of Clinical Laboratory, ${ }^{5}$ Department of Health Examination Center, Peking Union \\ Medical College Hospital, Chinese Academy of Medical Sciences, Beijing 100730, China \\ Contributions: (I) Conception and design: H Fu, B Peng; (II) Administrative support: None; (III) Provision of study materials or patients: B Peng, L \\ Qiu, S Lin; (IV) Collection and assembly of data: R Zhu, L Qiu, S Lin; (V) Data analysis and interpretation: H Fu, D Zhang, L Cui, B Peng; (VI) \\ Manuscript writing: All authors; (VII) Final approval of manuscript: All authors. \\ Correspondence to: Bin Peng. Department of Neurology, Peking Union Medical College Hospital, Chinese Academy of Medical Sciences, No.1 \\ Shuaifuyuan, Dongcheng District, Beijing 100730, China. Email: pengbin3@hotmail.com; Ling Qiu. Department of Clinical Laboratory, Peking \\ Union Medical College Hospital, Chinese Academy of Medical Sciences, No. 1 Shuaifuyuan, Dongcheng District, Beijing 100730, China. \\ Email: lingqiubj@aliyun.com; Songbai Lin. Department of Health Examination Center, Peking Union Medical College Hospital, Chinese Academy \\ of Medical Sciences, No. 1 Shuaifuyuan, Dongcheng District, Beijing 100730, China. Email: linsb@pumch.cn.
}

Background: Lipoprotein(a) $[\mathrm{Lp}(\mathrm{a})]$ is a risk factor of coronary heart disease, however, its effects on stroke are less well-defined.

Methods: We performed a single-center, retrospective case-control study in 1,953 and 196 ischemic stroke and hemorrhagic stroke in-hospital patients, respectively. Controls were healthy individuals that were matched for sex and age ( \pm 5 years) for the ischemic (1:1 ratio) and hemorrhagic (1:2 ratio) stroke. $\mathrm{Lp}$ (a) concentration was measured using the latex agglutination turbidimetric method. Logarithmic transformation and quartile categorization were applied to adjust for the skewed distribution of $\mathrm{Lp}$ (a). Conditional logistic regression models were used to assess the association between $\mathrm{Lp}(\mathrm{a})$ and stroke risk.

Results: Median Lp(a) concentration was higher in stroke patients when compared with controls (12.2 vs. $8.60 \mathrm{mg} / \mathrm{dL})$ and hemorrhagic strokes (14.40 vs. $13.40 \mathrm{mg} / \mathrm{dL})$. The conditional multivariate analysis revealed a positive association between $\mathrm{Lp}(\mathrm{a})$ and ischemic stroke $(\mathrm{OR}=2.03,2.36$, and 2.03 for quartiles 2, 3 and 4, respectively, $v s$. quartile $1 ; \mathrm{P}<0.001)$. In addition, elevated $\mathrm{Lp}$ (a) was also significantly associated with increased hemorrhagic stroke risk, after adjusted for potential covariates ( $\mathrm{OR}=1.93,3.24$, and 2.19 for quartile 2, 3 and 4 respectively vs. quartile $1, \mathrm{P}<0.05)$. The stratified analyses for ischemic and hemorrhagic stroke revealed significant association between elevated log-transformed $\mathrm{Lp}(\mathrm{a})$ and ischemic stroke in men. Furthermore, there was a trend towards a higher stroke risk for younger patients compared with older patients.

Conclusions: Elevated serum $\mathrm{Lp}(\mathrm{a})$ is significantly positively correlated with ischemic and hemorrhagic stroke risk in the Chinese Han population, especially among men and younger patients.

Keywords: Lipoprotein(a) [Lp(a)]; ischemic stroke; hemorrhagic stroke; case-control study

Submitted Sep 30, 2019. Accepted for publication Dec 03, 2019.

doi: $10.21037 /$ atm.2020.01.38

View this article at: http://dx.doi.org/10.21037/atm.2020.01.38 


\section{Introduction}

Stroke has become the second leading cause of disease burden worldwide and the first leading cause of disability among Chinese populations (1). Chinese populations have a higher overall incidence of stroke and greater proportion of hemorrhagic stroke than Caucasian populations. Despite a greater incidence of ischemic stroke, hemorrhagic stroke contributes to a similar number of deaths in China (2). Conventional risk factors include hypertension, diabetes, and smoking; however, approximately $25 \%$ of all ischemic strokes lack a clear cause (3). Therefore, it is important to identify other potential stroke risk factors.

Lipoprotein (a) $[\mathrm{Lp}(\mathrm{a})]$ is formed from a low-density lipoprotein (LDL)-like particle and the glycoprotein, apolipoprotein(a) [apo(a)], linked to apolipoprotein B in the LDL by a single disulphide bond (4). Lp(a) plays a role in atherogenic and pro-thrombotic/anti-fibrinolytic processes $(5,6)$. In addition, studies have reported roles in inflammatory responses, binding of oxidized phospholipids, and vascular remodeling (7).

A causal association between elevated $\mathrm{Lp}(\mathrm{a})$ and increased risk of myocardial infarction has been proved (8); however, there are conflicting reports regarding the relationship between stroke risk and $\mathrm{Lp}$ (a) concentration. Several metaanalyses with large samples have shown that $\mathrm{Lp}(\mathrm{a})$ is a risk factor for ischemic stroke (9-11). However, a systematic literature review with 16 prospective studies found no significant effect of $\mathrm{Lp}$ (a) on stroke risk (12). Additionally, the concentration of serum $\mathrm{Lp}(\mathrm{a})$ is determined by genetic factors primarily and differs substantially across ethnicities. Few studies have assessed the effects of $L p(a)$ on stroke in Asian populations. Moreover, previous studies have used unclassified stroke or ischemic stroke patients only; fewer studies have assessed the effect of $\mathrm{Lp}(\mathrm{a})$ on hemorrhagic stroke. Therefore, we sought to detect the association between $\mathrm{Lp}(\mathrm{a})$ and ischemic or hemorrhagic stroke risk in this case-control study in the Chinese Han population.

\section{Methods}

\section{Study participates}

This was a retrospective case-control study assessing the relationship between serum $\mathrm{Lp}(\mathrm{a})$ and ischemic and hemorrhagic stroke risk in the Chinese Han population. Cases were extracted from administrative data from the Peking Union Medical College Hospital (PUMCH), a national comprehensive hospital. Patients $>18$ years old were eligible for inclusion if they had been diagnosed with ischemic (International Classification of Diseases, 10th edition (ICD-10, code I63) or hemorrhagic stroke (ICD-10; codes I61, I62) between January 1, 2009 and December 31, 2018. Patients with transient ischemic attack were excluded.

All diagnostic information and laboratory data were obtained from discharge files and the hospital's electronic laboratory database. Exclusion criteria were non-Han nationality, malignant tumor with $\leq 5$ years remission, chronic liver disease ( $>3$-fold transaminase elevation), chronic kidney disease (serum creatinine, $\geq 152.5 \mu \mathrm{mol} / \mathrm{L}$ ), and systemic autoimmune disease. For patients with multiple hospitalizations, we included records during hospitalization for the first stroke diagnosis only. Eligible healthy individuals without stroke history were selected from the health examination center of PUMCH during the same period with the same exclusion criteria. Control participants were selected and matched by age $( \pm 5$ years $)$ and gender for the ischemic stroke (1:1 ratio) and hemorrhagic (1:2 ratio) group, respectively. The study protocol was approved by the Ethics Committee of the PUMCH who waived the requirement for informed consent because this study used anonymized data in a retrospective analysis.

Hypertension was defined as the use of antihypertensive drugs or with a diagnosis in the medical records. Diabetes (DM) was diagnosed when the percentage of hemoglobin A1c (HbA1c\%) was $>6.5 \%$, use of glucose-lowering drugs, or with a diagnosis in the medical records. Coronary atherosclerotic heart disease (CAD) refers to those with a record of coronary atherosclerotic stenosis or occlusion, or coronary stent implantation. Hyperlipidemia was defined as LDL $>4.1 \mathrm{mmol} / \mathrm{L}, \mathrm{HDL}<1.0 \mathrm{mmol} / \mathrm{L}$, or the use of lipidlowering medication (13).

\section{Lp(a) measurement}

$\mathrm{Lp}(\mathrm{a})$ and other plasma biochemical parameters were analyzed in the Department of Laboratory Medicine at PUMCH and archived data were identified using computer retrieval. Blood samples were obtained after a 12-hour overnight fast and centrifuged at 3,500 r/min for $15 \mathrm{~min}$ after standing for $30 \mathrm{~min}$. The concentration of serum $\mathrm{Lp}$ (a) was measured using the latex agglutination turbidimetric method with mouse monoclonal anti-Lp(a) antibody (Beckman AU5800 automatic biochemistry, Sekisui Medical Company, Tokyo, Japan). The detection range was $0-100$ $\mathrm{mg} / \mathrm{dL}$ with a normal value of below $30 \mathrm{mg} / \mathrm{dL}$. The intra- and inter-assay coefficients of variation $(\mathrm{CV})$ were 
below $5.0 \%$ and $10 \%$, respectively. Triglyceride (TG), total cholesterol (TC), LDL cholesterol (LDL-c), and homocysteine (HCY) were measured using an enzymatic method. High density lipoprotein cholesterol (HDL-c) was determined by direct methods. Hypersensitive C-reactive protein (hsCRP) was detected using immune turbidimetric analysis. All plasma biochemical parameters were assayed by an automatic biochemistry analyzer (AU5800, Beckman Coulter, Brea, CA).

\section{Statistical analysis}

Data are presented as mean \pm standard deviation (SD) for continuous normally distributed variables and median with interquartile ranges for skewed distributed variables. Student's $t$-test was applied to compare normally distributed variables or skewed distributed variables after logarithm transformation, and Pearson's $\chi^{2}$ test was applied for categorical comparisons. A common logarithm ( $\mathrm{Lg}$ ) transformation was applied for the skewed distribution of $\mathrm{Lp}(\mathrm{a})$. Further, analysis was undertaken to assess the relationship between quartiles of Lg-transformed $\mathrm{Lp}$ (a) levels and stroke risk. Univariate and multivariate conditional logistic regression models for matched casecontrol data were used to calculate the odds ratios (ORs) and $95 \%$ confidence intervals ( $95 \% \mathrm{CI}$ ) of $\mathrm{Lg}$-transformed $\mathrm{Lp}(\mathrm{a})$ as a categorical variable. We adjusted for diabetes, hyperlipidemia, and hypersensitive CRP. In addition, we also adjusted for variables that changed the matched odds ratio by at least $10 \%$ when added to this model. Interaction and stratified analyses were conducted by age $(<55$ and $\geq 55$ years) and sex. A two-tailed $P$ value of $<0.05$ was considered significant. All statistical analyses were performed with R software, EmpowerStats (X\&Y Solutions, Boston, MA) and SAS 9.4 (SAS Institute Inc., Cary, NC).

\section{Results}

\section{Clinical characteristics}

Among the 464,567 hospitalizations in the study cohort, we enrolled 2,296 and 205 ischemic and hemorrhagic stroke patients, respectively, based on the inclusion and exclusion criteria (Figure S1). We randomly selected 1,953 and 392 age- and sex-matched healthy controls for the ischemic and hemorrhagic groups, respectively. The characteristics of the controls are presented in Table 1. The ischemic group had a higher mean age and higher proportion of males compared with the hemorrhagic group. The proportions of DM and CAD were higher in the ischemic group. The median $\mathrm{Lp}$ (a) concentration in the two groups was similar (Table S1). Cases and controls were similar in age, sex and BMI in the ischemic group. There was a greater burden of hypertension, diabetes, cardiovascular disease, and hyperlipidemia, in ischemic stroke cases compared with controls. In addition, data showed lower median HDL, LDL, total cholesterol, and triglyceride levels and higher homocysteine levels in ischemic stroke cases when compared with controls. A similar distribution was found in the hemorrhagic group, except that the hemorrhagic cases showed lower CAD ratios and higher triglyceride levels when compared with controls.

\section{Lp(a) and ischemic stroke risk}

The median concentration of $\mathrm{Lp}$ (a) was 12.2 and $8.60 \mathrm{mg} / \mathrm{dL}$ in the ischemic stroke and control groups, respectively (Table 1). We used conditional logistic regression models to assess whether $\mathrm{Lp}(\mathrm{a})$ was independently associated with ischemic stroke (Table 2). After adjustment for hypertension, diabetes, hyperlipidemia, and hsCRP, we found that lg-transformed Lp(a) was significantly associated with ischemic stroke with odd radios (ORs) and $95 \%$ confidence intervals (CI) for $\mathrm{lg}$ transformed $\mathrm{Lp}$ (a) quartiles 4,3 , and 2 (vs. quartile 1 as a reference) of $2.03(1.61,2.57), 2.36(1.85,2.99)$ and 2.03 $(1.60,2.59)$, respectively. After further adjustment for BMI, cardiovascular disease, and homocysteine on Model 2, the relationship persisted (all $\mathrm{P}$ values for the log-transformed $\mathrm{Lp}(\mathrm{a})$ quartiles 2,3 , and $4 v s$. quartile 1 were $<0.001$ ). In the stratified analysis, we found a significant association between elevated $\mathrm{Lg}$-transformed $\mathrm{Lp}(\mathrm{a})$ and ischemic stroke in men. No statistically significant association was found in women in the Model adjusted for hypertension, hyperlipidemia, and hsCRP; and there was a significant interaction $(\mathrm{P}=0.006)$. Interestingly, younger patients ( $<55$ years) showed greater increased risk than older patients for ischemic stroke in the multivariable analysis, and no significant differences for interactions were detected (Figure 1).

\section{Lp(a) and hemorrhagic stroke risk}

There was a higher median $\mathrm{Lp}(\mathrm{a})$ concentration in the hemorrhagic group $(14.40 \mathrm{mg} / \mathrm{dL})$ compared with the control group $(13.40 \mathrm{mg} / \mathrm{dL}$; Table 1$)$. The association between log-transformed $\mathrm{Lp}(\mathrm{a})$ and hemorrhagic stroke 
Table 1 Baseline characteristics of ischemic and hemorrhagic stroke cases and controls after age and sex matching

\begin{tabular}{|c|c|c|c|c|}
\hline Characteristics & \multicolumn{2}{|c|}{ Ischemic stroke } & \multicolumn{2}{|c|}{ Hemorrhagic stroke } \\
\hline Age, years & $62.38 \pm 11.82$ & $59.97 \pm 11.07$ & $57.90 \pm 13.78$ & $57.91 \pm 13.76$ \\
\hline Male, \% & 64.98 & 64.98 & 55.61 & 55.61 \\
\hline BMI, $\mathrm{kg} / \mathrm{m}^{2}$ & $25.21 \pm 3.67$ & $25.22 \pm 3.24$ & $24.46 \pm 3.64$ & $24.74 \pm 3.29$ \\
\hline DM, \% & 39.99 & 18.23 & 22.45 & 15.31 \\
\hline $\mathrm{CAD}, \%$ & 23.70 & 18.23 & 12.8 & 17.34 \\
\hline Hyperlipidemia, \% & 68.00 & 33.29 & 62.76 & 30.61 \\
\hline $\mathrm{TC}, \mathrm{mmol} / \mathrm{L}$ & 4.09 (3.41, 4.92) & $4.82(4.17,5.49)$ & $4.2(3.77,5.28)$ & $4.82(4.27,5.41)$ \\
\hline LDL-c, mmol/L & $2.41(1.83,3.07)$ & $3.07(2.48,3.63)$ & $2.71(2.16,3.37)$ & $3.08(2.56,3.57)$ \\
\hline $\mathrm{Lp}(\mathrm{a}), \mathrm{mg} / \mathrm{dL}$ & $12.20(5.80,24.80)$ & $8.60(3.70,21.30)$ & $14.40(11.75,18.45)$ & $13.40(11.30,16.10)$ \\
\hline $\mathrm{HCY}, \mu \mathrm{mol} / \mathrm{L}$ & $14.20(11.40,18.65)$ & $13.30(11.50,15.67)$ & $13.05(7.25,25.33)$ & $9.70(3.98,24.00)$ \\
\hline hsCRP, mg/dL & $1.74(0.68,4.75)$ & $0.80(0.44,1.66)$ & $3.26(1.10,14.71)$ & $0.77(0.40,1.58)$ \\
\hline
\end{tabular}

Continuous variables presented as mean \pm standard deviation or median (Q1, Q3).

Table 2 Odd ratios (95\% confidence intervals) for ischemic stroke and lg-transformed $\mathrm{Lp}$ (a) concentration

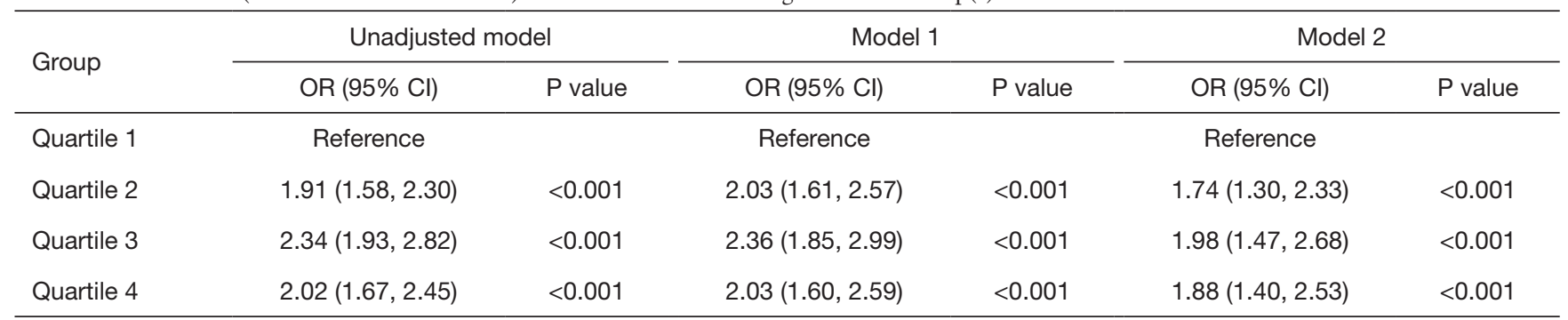

Model 1: adjusted for hypertension, diabetes, hyperlipidemia, hypersensitive C-reactive protein. Model 2: adjusted for body mass index, diabetes, hypertension, cardiovascular disease, hyperlipidemia, hypersensitive C-reactive protein, homocysteine. The $25^{\text {th }}, 50^{\text {th }}$, and $75^{\text {th }}$ percentile cut-off points for corresponding $\mathrm{Lp}(\mathrm{a})$ are $4.6,10.3$, and $23.2 \mathrm{mg} / \mathrm{dL}$, respectively.

was assessed using conditional logistic regression models (Table 3). Compared with patients in the lowest quartile, the ORs and $95 \%$ CIs for log-transformed $\mathrm{Lp}$ (a) quartiles $2-4$ were 1.88 (1.08, 3.12), $2.46(1.43,4.25)$ and 2.18 (1.28, $3.72)$, respectively, in the unadjusted model. Additional adjustment for hypertension, hyperlipidemia, and hsCRP yielded similar results $[\mathrm{OR}=1.93(1.02,3.67), 3.24$ (1.65, $6.39)$, and $2.19(1.12,4.29)$ for quartiles $2-4$, respectively, vs. the reference quartile $1, \mathrm{P}=0.043, \mathrm{P}<0.001$ and $\mathrm{P}=0.022]$. The correlation between $\mathrm{Lp}(\mathrm{a})$ level and the risk of hemorrhagic stroke also survived full adjustment for diabetes, hypertension, cardiovascular disease, hyperlipidemia, and hsCPR in Model 2. The stratified analysis that adjusted for hypertension, hyperlipidemia, and hsCPR was conducted according to sex and age. Similar to the ischemic stroke, elevated Lg-transformed $\mathrm{Lp}$ (a) concentration was associated with a higher risk of hemorrhagic stroke in men alone, after adjusting for potential confounding factors. Increased hemorrhagic stroke risk was associated with elevated Lg-transformed 

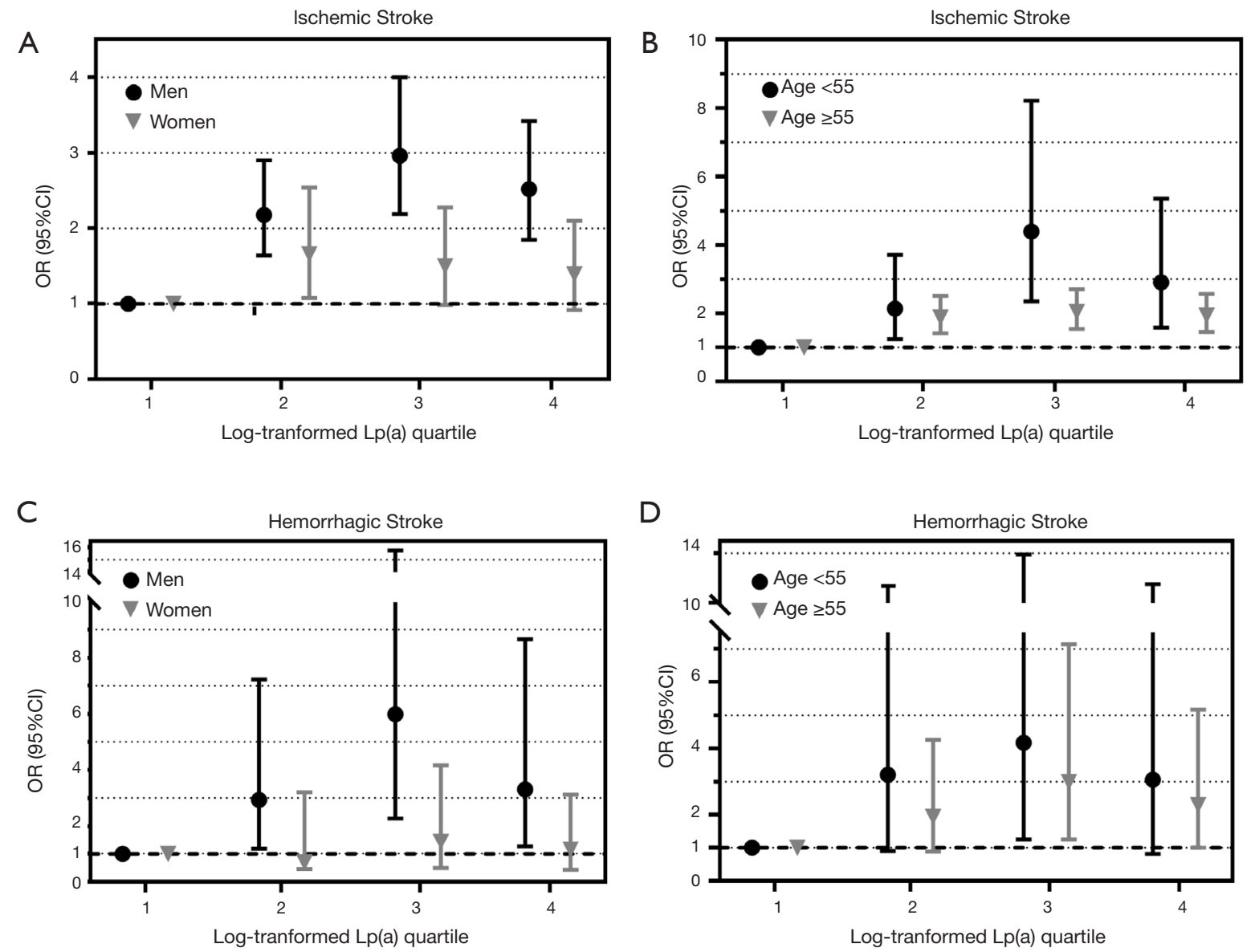

Figure 1 Odd ratios (95\% confidence intervals) for ischemic stroke and hemorrhagic stroke of log-transformed Lipoprotein (a) quartiles stratified by sex (A, C) and age (B, D). The model was adjusted for hypertension, hyperlipidemia, hypersensitive C-reactive protein.

$\mathrm{Lp}$ (a) in both younger ( $<55$ years) and older patients ( $\geq 55$ years), but the effects were smaller in the older group (Figure 1).

\section{Discussion}

In this retrospective case-control study, we identified the associations between elevated $\mathrm{Lp}$ (a) and ischemic or hemorrhagic stroke risk compared with the age- and gender-matched healthy controls. Furthermore, we found an increased stroke risk associated with $\mathrm{Lp}(\mathrm{a})$ among younger patients. Interestingly, the effect of elevated $L p(a)$ on both stroke types was significant among men alone. Surprisingly, LDL-c, TC, and TG levels were lower in the stroke groups when compared with the controls, which may be due to the higher proportion of dyslipidemia in the stroke group with a lower proportion and target value for lipid-lowering treatment.

The effect of serum $\mathrm{Lp}$ (a) level on the risk of coronary heart disease is well-established; however, the effects on stroke risk remain controversial. Our results correspond with several previous studies on ischemic stroke. A metaanalysis of 126,634 individuals by the Emerging Risk Factors Collaboration demonstrated a significantly higher risk for ischemic stroke with a risk ratio (RR) of $1.10(95 \%$ CI: 1.02-1.18) per 3.5-fold higher Lp(a) concentrations (9). However, $>90 \%$ of the participants in this study were of European continental ancestry that attenuated the generalization to patients of all ethnicities. Nave et al. have summarized 20 articles comprising 90,904 cases and concluded that $\mathrm{Lp}(\mathrm{a})$ might be an independent risk factor for ischemic stroke with a pooled OR of 1.41 (95\% CI: 1.26-1.57) for case-control studies and 1.29 (95\% CI: $1.06-$ 1.58 ) for prospective studies (10). In contrast, data from 
Table 3 Odd ratios (95\% confidence intervals) for hemorrhagic stroke and lg-transformed Lp(a) concentration

\begin{tabular}{|c|c|c|c|c|c|c|}
\hline Group & \multicolumn{2}{|c|}{ Unadjusted model } & \multicolumn{2}{|c|}{ Model 1} & \multicolumn{2}{|c|}{ Model 2} \\
\hline Quartile 1 & Reference & & Reference & & Reference & \\
\hline Quartile 2 & $1.88(1.08,3.12)$ & 0.026 & $1.93(1.02,3.67)$ & 0.043 & $2.06(1.08,3.93)$ & 0.029 \\
\hline Quartile 3 & $2.46(1.43,4.25)$ & 0.001 & $3.24(1.65,6.39)$ & $<0.001$ & $3.30(1.66,6.56)$ & $<0.001$ \\
\hline
\end{tabular}

Model 1: adjusted for hypertension, hyperlipidemia, hypersensitive C-reactive protein. Model 2: adjusted for diabetes, hypertension, cardiovascular disease, hyperlipidemia, hypersensitive C-reactive protein. $25^{\text {th }}, 50^{\text {th }}$, and $75^{\text {th }}$ percentile cut-off points for corresponding $\mathrm{Lp}(\mathrm{a})$ are $4.5,11.2$, and $24.3 \mathrm{mg} / \mathrm{dL}$, respectively.

the China Kadoorie Biobank (CKB) nested case-control study showed no significant association between $\mathrm{Lp}(\mathrm{a})$ and ischemic stroke in a sample of 32,869 incident ischemic stroke cases (14). It should be noted that the female rate in the ischemic group was higher in the CKB study, which might reduce the intergroup differences because of gender differences of the association between $\mathrm{Lp}(\mathrm{a})$ and ischemic stroke. Overall, the different conclusions of studies assessing $\mathrm{Lp}(\mathrm{a})$ and ischemic stroke risk might be due to differences in study design, definition of vascular end points, and participant sampling. Another factor related to the discrepancy between these results is the long-term storage of samples, since the $\mathrm{Lp}$ (a) measurement could be less reliable if samples are stored for longer than 6 weeks (4).

Mechanisms underlying the effect of $\mathrm{Lp}(\mathrm{a})$ on vascular diseases remain unclear and require further research. Nevertheless, the number of studies that have explored the correlation between ischemic subtypes and $\mathrm{Lp}(\mathrm{a})$ is limited. It has been reported that serum $\mathrm{Lp}(\mathrm{a})$ is significantly higher in large-artery atherosclerosis compared with other stroke subtypes $(15,16)$. Additionally, the association between large-artery atherosclerosis subtypes of ischemic stroke and $\mathrm{Lp}(\mathrm{a})$ has been supported by genetic variant analysis of the $\mathrm{Lp}$ (a) related gene, $L P A$ (4). However, we were unable to stratify our patient group by ischemic stroke subtypes due to limitations in our data collection.

Most previous studies have focused on ischemic stroke or unclassified stroke. Our study also investigated the effect of $\mathrm{Lp}(\mathrm{a})$ on hemorrhagic stroke; however, the literature offers mixed results, ranging from negative to positive associations between $\mathrm{Lp}(\mathrm{a})$ and hemorrhagic stroke. Consistent with our results, a multicenter case-control study in Chinese patients with 499 events found increased $\mathrm{Lp}$ (a) levels led to a 1.64fold increase (95\% CI: 1.21-2.21) in the risk of hemorrhagic stroke (17). In contrast, results from a population-based prospective cohort study with 84 hemorrhagic stroke events suggest that decreased $\mathrm{Lp}(\mathrm{a})$ may be a predictive marker for future cerebral hemorrhage (18). A different study has reported no significant association between $L p(a)$ levels and hemorrhagic stroke (14). Furthermore, the Emerging Risk Factors Collaboration concluded that there was no significant association between $\mathrm{Lp}(\mathrm{a})$ and hemorrhagic stroke (adjusted RR: 1.06, 95\% CI: 0.90-1.26) (9). The limited number of hemorrhagic events included in these studies might be a contributing factor for the inconsistent results. In addition, the causes of cerebral hemorrhage are varied and no study has detected a relationship with the stratification of hemorrhagic types, which might be another contributing factor.

In this study, we observed that the concentration of $\mathrm{Lp}$ (a) had a greater effect on ischemic and hemorrhagic stroke in men. The same trend was also observed in previous studies assessing ischemic stroke $(10,19)$. This might be due to genetically predetermined differences in $\mathrm{Lp}$ (a) levels between sexes. Moreover, most studies did not adjust for differences in living habits, including smoking and dietary habits between the sexes. Studies that take into account sex differences in the association between $L p(a)$ and hemorrhagic stroke are lacking. Nonetheless, consistent with previous findings, we found a more pronounced association between $\mathrm{Lp}(\mathrm{a})$ and stroke risk in younger patients (10). A meta-analysis of four case-control studies also showed a positive association between elevated $\mathrm{Lp}(\mathrm{a})$ and stroke in children (OR: 4.24, 95\% CI: 2.94-6.11) (20). This finding might be due to a lower absolute risk in younger patients was lower. Additionally, $\mathrm{Lp}$ (a) levels are closely linked to genetic factors (4), while environmental risk factors play an increasingly important role in stroke 
with age. Thus, $\mathrm{Lp}(\mathrm{a})$ might be a potential risk factor for stroke in young adults.

Compared with observational studies, studies assessing whether the therapeutic lowering of $\mathrm{Lp}$ (a) reduces stroke risk could provide powerful evidence. However, this is not easy because effective, targeted Lp(a)-lowering therapies are limited. The currently available therapies for lowering $\mathrm{Lp}(\mathrm{a})$ include lipid apheresis, niacin, inhibitors of proprotein convertase subtilisin/kexin type 9, and cholesteryl ester transfer protein inhibitors (21). Antisense oligonucleotides targeting Apo(a), including IONIS-APO(a)Rx and IONISAPO(a)LRx, have shown reductions in $\mathrm{Lp}$ (a) by $65 \%$ and 92-99\%, respectively (22). A further study selected patients with high $\mathrm{Lp}$ (a) levels, and reported that isolated lowering of $\mathrm{Lp}(\mathrm{a})$ could lower the incidence rate of cardiovascular events (23). Further prospective studies are required to evaluate this therapeutic effect on stroke risk.

There are several limitations in our study. Firstly, as a case-control study, the results can only show associations between $\mathrm{Lp}$ (a) levels and stroke; however, and they do not allow any assessment of causality. Secondly, although the concentration of $\mathrm{Lp}(\mathrm{a})$ is relatively stable throughout life (24), its concentration collected after a stroke may not accurately reflect pre-stroke exposure. Thirdly, the time intervals between $\mathrm{Lp}(\mathrm{a})$ measurement and stroke were differed between individuals. We were also limited by the selection of controls, who were recruited from the health examination center rather than a population-based sample. Fourthly, studies have reported that statins increase $\mathrm{Lp}(\mathrm{a})$ levels (25), which could be a bias of this study. Lastly, this study did not analyze the effect of $\mathrm{Lp}$ (a) concentration on the risk of different ischemic stroke types according to the Trial of Org 10172 in Acute Stroke Treatment (TOAST), which might diminish the actual absolute risk and limit the detection of a pathogenic mechanism for $\mathrm{Lp}(\mathrm{a})$.

In conclusion, our study found that the concentration of serum $\mathrm{Lp}(\mathrm{a})$ was independently associated with both ischemic and hemorrhagic stroke risk, especially among younger individuals in a Chinese Han population. Further, this effect was only significant among men. A powerful and specific $\mathrm{Lp}$ (a) lowering method is required to demonstrate causality and guide methods of stroke prevention.

\section{Acknowledgments}

Funding: This study was supported by the Chinese Academy of Medical Sciences (CAMS) Innovation Fund for Medical
Sciences (CIFMS), Grant Number: 2016-I2M-1-002 .

\section{Footnote}

Conflicts of Interest: The authors have no conflicts of interest to declare.

Ethical Statement: The authors are accountable for all aspects of the work in ensuring that questions related to the accuracy or integrity of any part of the work are appropriately investigated and resolved. The study protocol was approved by the Ethics Committee of the PUMCH who waived the requirement for informed consent because this study used anonymized data in a retrospective analysis.

Open Access Statement: This is an Open Access article distributed in accordance with the Creative Commons Attribution-NonCommercial-NoDerivs 4.0 International License (CC BY-NC-ND 4.0), which permits the noncommercial replication and distribution of the article with the strict proviso that no changes or edits are made and the original work is properly cited (including links to both the formal publication through the relevant DOI and the license). See: https://creativecommons.org/licenses/by-nc-nd/4.0/.

\section{References}

1. GBD 2016 DALYs and HALE Collaborators. Global, regional, and national disability-adjusted life-years (DALYs) for 333 diseases and injuries and healthy life expectancy (HALE) for 195 countries and territories, 1990-2016: a systematic analysis for the Global Burden of Disease Study 2016. Lancet 2017;390:1260-344.

2. Tsai CF, Thomas B, Sudlow CL. Epidemiology of stroke and its subtypes in Chinese vs white populations: a systematic review. Neurology 2013;81:264-72.

3. Hart RG, Diener HC, Coutts SB, et al. Embolic strokes of undetermined source: the case for a new clinical construct. Lancet Neurol 2014;13:429-38.

4. Kronenberg F. Human Genetics and the Causal Role of Lipoprotein(a) for Various Diseases. Cardiovasc Drugs Ther 2016;30:87-100.

5. Vogt A. Lipoprotein(a)-apheresis in the light of new drug developments. Atheroscler Suppl 2017;30:38-43.

6. Cho T, Romagnuolo R, Scipione C, et al. Apolipoprotein(a) stimulates nuclear translocation of $\beta$-catenin: a novel pathogenic mechanism for lipoprotein(a). Mol Biol Cell 
2013;24:210-21.

7. Riches K, Porter KE. Lipoprotein(a): Cellular Effects and Molecular Mechanisms. Cholesterol. 2012;2012:923289.

8. Kamstrup PR, Tybjaerg-Hansen A, Steffensen R, et al. Genetically elevated lipoprotein(a) and increased risk of myocardial infarction. JAMA 2009;301:2331-9.

9. Emerging Risk Factors Collaboration, Erqou S, Kaptoge S, et al. Lipoprotein(a) concentration and the risk of coronary heart disease, stroke, and nonvascular mortality. JAMA 2009;302:412-23.

10. Nave AH, Lange KS, Leonards CO, et al. Lipoprotein (a) as a risk factor for ischemic stroke: a meta-analysis. Atherosclerosis 2015;242:496-503.

11. Zhang J, Du R, Peng K, et al. Serum lipoprotein (a) is associated with increased risk of stroke in Chinese adults: A prospective study. Atherosclerosis. 2019;289:8-13.

12. Genser B, Dias KC, Siekmeier R, et al. Lipoprotein (a) and risk of cardiovascular disease--a systematic review and meta analysis of prospective studies. Clin Lab 2011;57:143-56.

13. Beheshtian A, Shitole SG, Segal AZ, et al. Lipoprotein (a) level, apolipoprotein (a) size, and risk of unexplained ischemic stroke in young and middle-aged adults. Atherosclerosis 2016;253:47-53.

14. Sun L, Clarke R, Bennett D, et al. Causal associations of blood lipids with risk of ischemic stroke and intracerebral hemorrhage in Chinese adults. Nat Med 2019;25:569-74.

15. Hong XW, Wu DM, Lu J, et al. Lipoprotein (a) as a Predictor of Early Stroke Recurrence in Acute Ischemic Stroke. Mol Neurobiol 2018;55:718-26.

16. Aronis KN, Zhao D, Hoogeveen RC, et al. Associations of Lipoprotein(a) Levels With Incident Atrial Fibrillation and Ischemic Stroke: The ARIC (Atherosclerosis Risk in Communities) Study. J Am Heart Assoc 2017;6. doi: 10.1161/JAHA.117.007372.

Cite this article as: Fu H, Zhang D, Zhu R, Cui L, Qiu L, Lin S, Peng B. Association between lipoprotein(a) concentration and the risk of stroke in the Chinese Han population: a retrospective case-control study. Ann Transl Med 2020;8(5):212. doi: 10.21037/atm.2020.01.38
17. Sun L, Li Z, Zhang H, et al. Pentanucleotide TTTTA repeat polymorphism of apolipoprotein(a) gene and plasma lipoprotein(a) are associated with ischemic and hemorrhagic stroke in Chinese: a multicenter case-control study in China. Stroke 2003;34:1617-22.

18. Ishikawa S, Kotani K, Kario K, et al. Inverse association between serum lipoprotein(a) and cerebral hemorrhage in the Japanese population. Thromb Res 2013;131:e54-8.

19. Boden-Albala B, Kargman DE, Lin IF, et al. Increased stroke risk and lipoprotein(a) in a multiethnic community: the Northern Manhattan Stroke Study. Cerebrovasc Dis 2010;30:237-43.

20. Sultan SM, Schupf N, Dowling MM, et al. Review of lipid and lipoprotein(a) abnormalities in childhood arterial ischemic stroke. Int J Stroke 2014;9:79-87.

21. Ferretti G, Bacchetti T, Johnston TP, et al. Lipoprotein(a): A missing culprit in the management of atherothrombosis?. J Cell Physiol 2018;233:2966-81.

22. Viney NJ, van Capelleveen JC, Geary RS, et al. Antisense oligonucleotides targeting apolipoprotein(a) in people with raised lipoprotein(a): two randomised, doubleblind, placebo-controlled, dose-ranging trials. Lancet 2016;388:2239-53.

23. Leebmann J, Roeseler E, Julius U, et al. Lipoprotein apheresis in patients with maximally tolerated lipidlowering therapy, lipoprotein(a)-hyperlipoproteinemia, and progressive cardiovascular disease: prospective observational multicenter study. Circulation 2013;128:2567-76.

24. Langsted A, Kamstrup PR, Nordestgaard BG. Lipoprotein(a): fasting and nonfasting levels, inflammation, and cardiovascular risk. Atherosclerosis 2014;234:95-101.

25. Enkhmaa B, Berglund L. Statins and Lp(a): The plot thickens. Atherosclerosis 2019;289:173-5. 


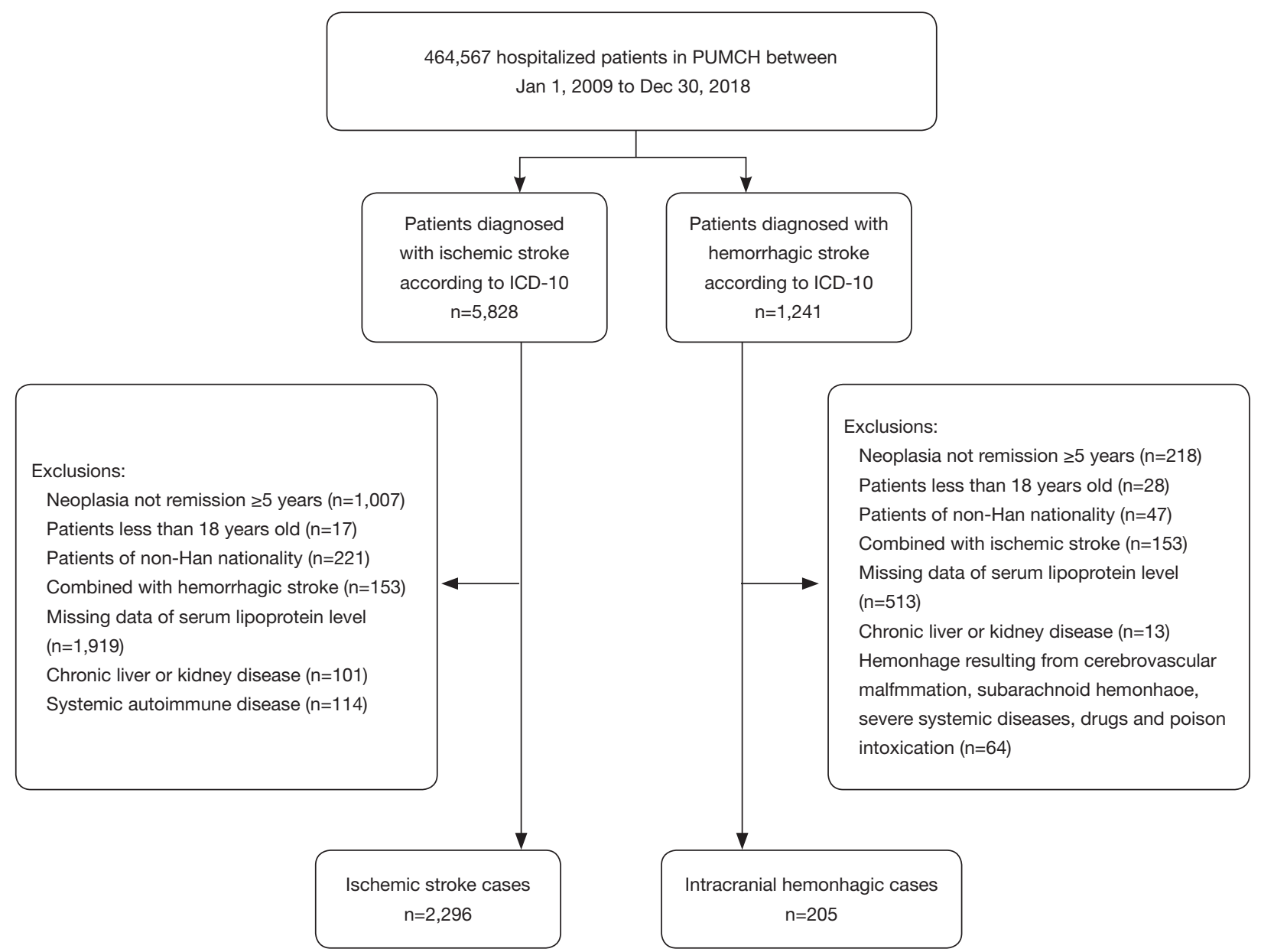

Figure S1 The data flow chart of the current study. 
Table S1 Comparation of characteristics between ischaemic group and hemorrhagic stroke group

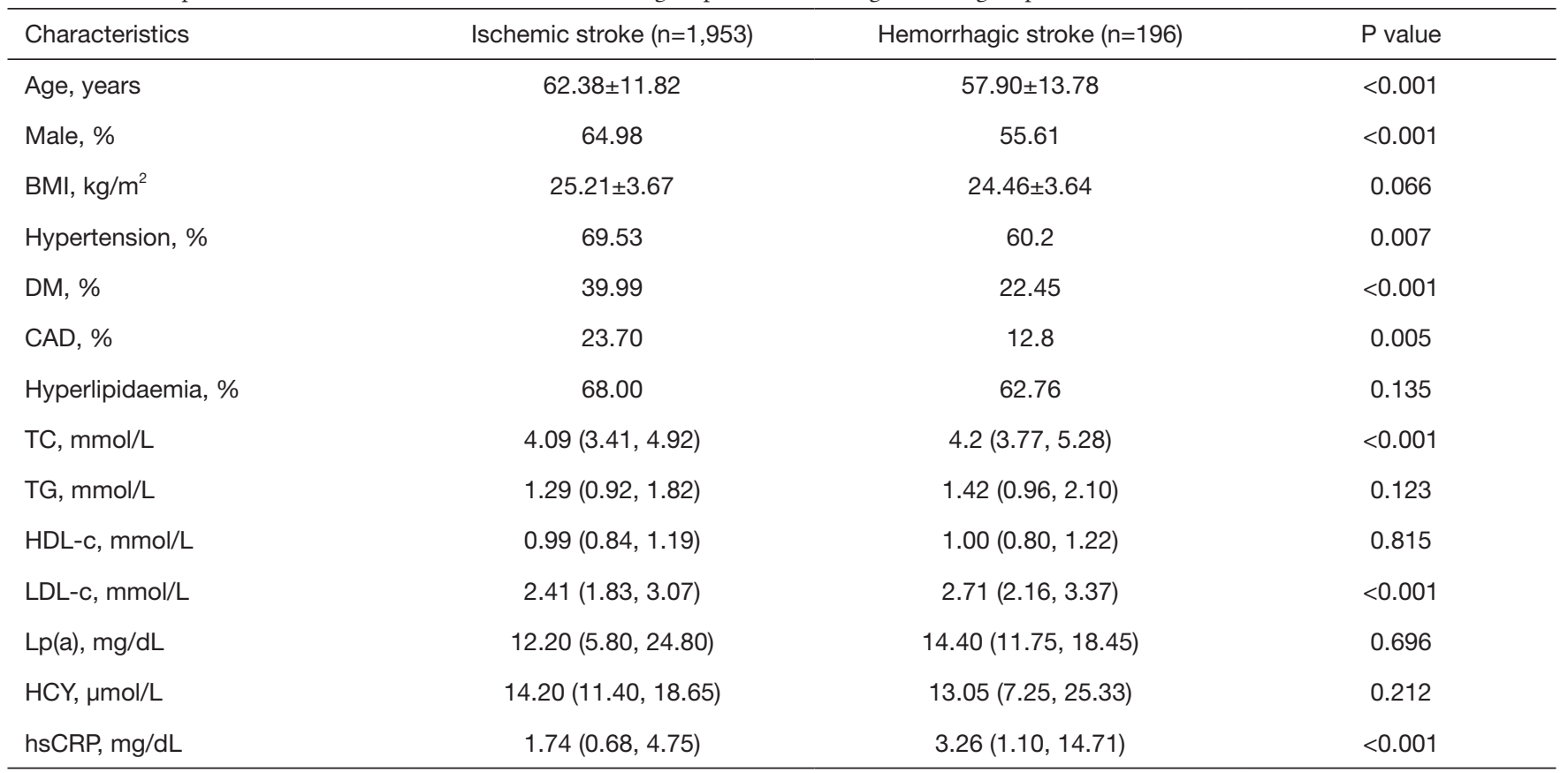

Continuous variables presented as mean \pm standard deviation or median (Q1, Q3). 